\title{
La intervención gerontológica en un establecimiento de asistencia social permanente privado: identificación de los beneficios que aporta a las personas mayores residentes
}

The gerontological intervention in a private permanent social assistance establishment: identification of the benefits it brings to the elderly residents

A intervenção gerontológica em um estabelecimento de assistência social permanente privada: identificação dos benefícios que traz para os idosos residentes

Jaqueline Guadalupe Guerrero Ceh Universidad Autónoma de Campeche, México jgguerre@uacam.mx https://orcid.org/0000-0002-2913-1309

Ana Rosa Can Valle Universidad Autónoma de Campeche, México anarocan@uacam.mx https://orcid.org/0000-0002-6849-9624

Lourdes Maricruz Morales Universidad Autónoma de Campeche, México al037294@uacam.mx https://orcid.org/0000-0002-4667-9173

\section{Resumen}

Introducción: México se encuentra en el camino hacia la toma de conciencia del reto que implica el envejecimiento de la población para las familias, la sociedad y los establecimientos de asistencia social permanente. Objetivo: Identificar los beneficios que aporta la intervención gerontológica en la salud de los residentes de un establecimiento privado de asistencia social permanente. Para ello, se ha planteado la siguiente hipótesis: después de una intervención gerontológica, los residentes se encuentran motivados para realizar actividad 
Revista Iberoamericana de las Ciencias de la Salud

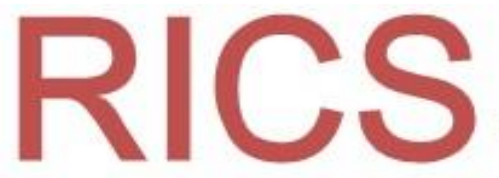

física, mejorar su postura y socializar sus experiencias. Método: El presente es un estudio con enfoque cuantitativo, de alcance descriptivo longitudinal, con un diseño experimental y de tipo cuasiexperimento. En concreto, se realizaron diez sesiones, cada una con dos horas de duración. Se tuvo una participación de ocho residentes de un establecimiento privado, con cierto nivel de dependencia. Resultados: La hipótesis planteada es aceptada, ya que todos los participantes demostraron mejoría en la flexibilidad, movilidad, socialización y aspecto cognitivo en los aspectos de atención y concentración. Conclusiones: Los beneficios que aporta la intervención gerontológica son satisfactorios para las personas residentes y para el establecimiento que ofrece sus servicios de asistencia social.

Palabras clave: establecimiento de asistencia social permanente, intervención gerontológica, persona mayor residente.

\section{Abstract}

Introduction Mexico is on the way to becoming aware of the challenge that the aging of the population implies for families, society and permanent social assistance establishments. Objective: Identify the benefits of gerontological intervention in the health of residents of a private permanent social assistance establishment. For this, the following hypothesis has been proposed: after a gerontological intervention, the residents are motivated to perform physical activity, improve their position and socialize their experiences. Method: The present is a study with a quantitative approach, of longitudinal descriptive scope, with an experimental design and of the quasi-experimental type. Specifically, ten sessions were held, each lasting two hours. There was participation of eight residents of a private establishment, with a certain level of dependency. Results: The proposed hypothesis is accepted, since all the participants demonstrated improvement in flexibility, mobility, socialization and cognitive aspect in attention and concentration aspects. Conclusions: The benefits provided by the gerontological intervention are satisfactory for the residents and for the establishment that offers its temporary social assistance services. 
Keywords: establishment of permanent social assistance, gerontological intervention, elderly person resident.

\section{Resumo}

Introdução: O México está a caminho de tomar consciência do desafio que o envelhecimento da população implica para as famílias, a sociedade e os estabelecimentos permanentes de assistência social. Objetivo: Identificar os benefícios da intervenção gerontológica na saúde de residentes de um estabelecimento privado de assistência social permanente. Para tanto, propôs-se a seguinte hipótese: após uma intervenção gerontológica, os moradores são motivados a realizar atividades físicas, melhorar sua posição e socializar suas experiências. Método: Trata-se de um estudo com abordagem quantitativa, com escopo descritivo longitudinal, com delineamento experimental e tipo quase-experimental. Especificamente, dez sessões foram realizadas, cada uma com duração de duas horas. Houve participação de oito moradores de um estabelecimento privado, com certo nível de dependência. Resultados: A hipótese proposta é aceita, uma vez que todos os participantes demonstraram melhora na flexibilidade, mobilidade, socialização e aspecto cognitivo nos aspectos atenção e concentração. Conclusões: Os benefícios proporcionados pela intervenção gerontológica são satisfatórios para os residentes e para o estabelecimento que oferece seus serviços de assistência social.

Palavras-chave: estabelecimento de assistência social permanente, intervenção gerontológica, idoso residente.

Fecha recepción: Marzo 2018

Fecha aceptación: Julio 2018 


\section{Introducción}

Gracias a los avances en la ciencia médica, la esperanza de vida en el siglo XXI se ha incrementado considerablemente, lo cual ha obligado a planificar actividades de ocupación y distracción para una sociedad envejecida y cada vez más numerosa. En efecto, según estimaciones de la Organización Mundial de la Salud (OMS, 2015), se prevé que para 2050 una de cada cinco personas tendrá más de 60 años de edad, de las cuales 80 \% vivirá en los países con ingresos bajos y medios, entre los que se puede ubicar México.

Estos datos son coherentes con lo reportado en la Encuesta Intercensal 2015 del Instituto Nacional de Estadística y Geografía (Inegi) (2015), donde se señala que 38 \% de la población de nuestro país son personas adultas mayores, cifra que según las proyecciones de dicha institución podría multiplicarse de forma exponencial en el próximo censo de población y vivienda que se realizará en el año 2020. Aunado a este pronóstico, se debe tomar en cuenta que el número de hogares unipersonales también tiende a elevarse en todos los estados de México (Inegi, 2003, 2017), por lo que es indispensable que las distintas instituciones responsables de atender esta situación se encuentren preparadas para ofrecer el mejor servicio de cuidado para los adultos mayores que lo requieran.

En tal sentido, el Sistema Nacional para el Desarrollo Integral de la Familia (SNDIF) puso en marcha en 2015 cuatro centros gerontológicos en el país con un modelo de intervención constituido sobre la base de cuatro fundamentos: prevención, atención integral, formación e investigación. El primero de ellos (prevención) tiene como objetivo específico "grupos sociales informados en medidas preventivas sobre el proceso de envejecimiento mediante la estrategia de la promoción de la geroprofilaxis" (Instituto de Mayores y Servicios Sociales [Imserso], 2015, p. 42); este propósito resulta esencial trabajarlo desde temprana edad para evitar o retardar la aparición de enfermedades no transmisible en las personas mayores.

El segundo fundamento (atención integral) procura fomentar en las personas adultas mayores "un estado físico, mental, social, emocional y espiritual optimizado de acuerdo a su 
proceso de envejecimiento" (Imserso, 2015, p. 42), pues se considera que el equilibrio de las dimensiones del ser humano es fundamental para el disfrute de la vida.

El tercer fundamento (formación) se enfoca en la capacitación de un "capital humano con conocimientos y actualización gerontológica" (Imserso, 2015, p. 43); para ello, se intentan promover conocimientos que sirvan de sustento para mejorar el bienestar tanto del personal como de los huéspedes, pues se trabaja no solo en una mejor formación de quienes atienden a las personas mayores, sino que también se brinda la oportunidad a los adultos para que adquieran nuevos conocimientos, incrementen su cultura y desarrollen determinada habilidad con el fin de impulsar una ocupación metacognitiva.

Por último, el cuarto fundamento (investigación) se centra en "lograr centros gerontológicos y casas hogar generadores de conocimientos científicos mediante la estrategia de coordinación de acciones dirigidas al desarrollo y producción tanto de información como conocimiento sobre la población objetivo" (Imserso, 2015, p. 43). Esta generación del conocimiento es fundamental para el avance de las acciones realizadas porque permite evaluar y diseñar estrategias para que los procesos mejoren continuamente en beneficio de una atención de calidad para las personas mayores. No obstante, vale destacar que esta situación difícilmente se materializa, ya que para desarrollar una investigación se debe ingresar a la institución para observar el fenómeno objeto de estudio, lo cual podría dejar en evidencia el trato que se ofrece a los huéspedes, así como las condiciones reales de la infraestructura del establecimiento.

La Organización de las Naciones Unidas (ONU) en 2015, propuso diecisiete objetivos para el desarrollo sostenible (ODS), la Vicesecretaria General de las Naciones Unidad, Amina J. Mohammed, expresa que: "Los ODS son mecanismos apropiados que permitirán a la población y a sus dirigentes de forma conjunta, participar en la búsqueda de consensos sociales y disminuir las brechas.” (ONU, 2015, párr. 1). Esta investigación ha sido motivada por dos los 17 objetivos del desarrollo sostenible, el No. 3 y 10, los cuales corresponden a los ejes de: salud y bienestar y reducción de las desigualdades, respectivamente; todo el trabajo que se realice con los grupos vulnerables es favorable para incidir en la reducción de las brechas sociales. 
Revista Iberoamericana

de las Ciencias de la Salud

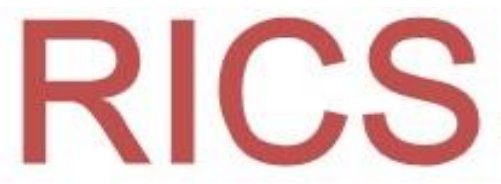

ISSN: $2395-8057$

Explicado todo lo anterior, se puede indicar que el objetivo del presente trabajo fue describir los beneficios que aporta la intervención gerontológica en la salud de los residentes de un establecimiento privado de asistencia social permanente. Antes de ello, sin embargo, a continuación se presenta un bosquejo en torno a las actividades que debe realizar un gerontólogo, las cuales se tomaron en cuenta en el desarrollo de esta propuesta.

\section{Actividades del gerontólogo}

El gerontólogo es un especialista que se encarga de estudiar el proceso de envejecimiento y de atender de forma integral a las personas mayores a través del desarrollo de programas gerontológicos de intervención. Al respecto, Mena et al. (2012) acota lo siguiente:

La importancia de las intervenciones gerontológicas radica en hacer efectivo el abordaje integral de la persona adulta mayor al reducir y prevenir situaciones de riesgos para la salud en su contexto bio-psico-social. Para poder desarrollar las intervenciones, el gerontólogo debe utilizar una serie de instrumentos especializados que aporten los datos necesarios para analizar y planear dichas intervenciones para la atención multidimensional de la persona adulta mayor (p. 8).

Efectivamente, el gerontólogo es el encargado de realizar la evaluación integral de la persona mayor a través de una historia clínica que se compone de los siguientes elementos: ficha de identificación, motivo de la atención, familiograma, valoración integral y clinimetría, lo cual constituye la primera parte. Después se integran trece escalas de valoración (doce enfocadas en la persona mayor y una en el familiar para identificar la presencia de sobrecarga del cuidador), las cuales se mencionan en seguida:

1. Apgar familiar.

2. Cuestionario de apoyo social percibido (DUKE-UNC)

3. Miniexamen del estado mental (minimental de Folstein).

4. Cuestionario del informante sobre deterioro cognoscitivo en la vejez (IQCODE). 
5. Escala de depresión geriátrica (GDS).

6. Índice de independencia en las actividades básicas de la vida diaria de Katz.

7. Índice de actividades instrumentales de la vida diaria de Lawton y Brody.

8. Escala mininutritional assessment de Guigoz y Velas (MNA).

9. Evaluación de estabilidad de la marcha y equilibrio (Tinetti).

10. Prueba levántate y anda (get up and go).

11. Cuestionario de actividades físicas de Nagi.

12. Investigación gerontológica del riesgo para el desarrollo del síndrome de caídas.

13. Cuestionario para la detección de sobrecarga del cuidador (Zarit y Zarit).

Como se puede apreciar, es evidente que la intervención gerontológica requiere tiempo, pues cada persona mayor es única, de ahí que sea indispensable considerar sus particularidades; aunado a esto, también se debe cultivar un ambiente de confianza para que la persona mayor coopere en las actividades que se intentan desarrollar.

La intervención gerontología, por ende, se encuentra integrada por cuatro fases: la primera se denomina historia clínica gerontológica, y es un espacio valioso en el que el gerontólogo interactúa con el paciente para ganar su confianza; la segunda es la aplicación de las trece escalas de valoración mencionadas; la tercera es el diagnóstico gerontológico, en el cual se analizan los datos obtenidos teniendo como herramienta la semiología; la cuarta etapa es la intervención gerontológica según el protocolo establecido para cada caso detectado en el diagnóstico. Sobre este particular, Sanjoaquín, Fernández, Mesa y García (2004) precisan lo siguiente:

La valoración geriátrica integral (VGI) surge, además, como respuesta a la alta prevalencia en el anciano de necesidades y problemas no diagnosticados, de disfunciones y dependencias reversibles no reconocidas, que se escapan a la valoración clínica tradicional (anamnesis y exploración física) (p. 59).

Esto significa que existen dos tipos de valoraciones: la clínica tradicional realizada por el médico y la enfermera, y la geriátrica integral efectuada por los gerontólogos, quienes conocen las escalas de valoración y pueden tener mejor acercamiento al diagnóstico y 
Revista Iberoamericana de las Ciencias de la Salud

determinar el tratamiento como resultado del trabajo con un equipo multidisciplinar del área de la salud.

En este sentido, Mena et al. (2012) sugiere veintiuna intervenciones que se pueden ajustar a cada situación diagnosticada:

1. Grupos de autoayuda o terapia cognoscitiva conductual.

2. Educación para la salud.

3. Estimulación sensorial.

4. Intervenciones indicadas para aprendizaje.

5. Intervenciones indicadas para lenguaje.

6. Intervenciones indicadas para orientación.

7. Intervenciones indicadas para memoria.

8. Intervenciones indicadas para evocación.

9. Intervenciones indicadas para cálculo.

10. Intervenciones indicadas para la construcción visoespacial.

11. Intervenciones indicadas para juicio.

12. Intervenciones indicadas para inteligencia.

13. Terapia ocupacional y estimulación física.

14. Ejercicios de calentamiento.

15. Intervenciones indicadas para alteraciones de la flexibilidad y elasticidad.

16. Intervenciones indicadas para la coordinación.

17. Intervenciones indicadas para la fuerza y resistencia muscular.

18. Intervenciones indicadas para ejercicios aeróbicos.

19. Intervenciones indicadas para hacer en el suelo o en la cama.

20. Intervenciones indicadas en sedestación

21. Intervenciones indicadas en cuidadores primarios.

En cada una de estas propuestas de intervención se mencionan las acciones que debe realizar el gerontólogo en coordinación con el grupo multidisciplinar. Asimismo, cabe destacar que una parte significativa de un programa de intervención gerontológica se encuentra integrada por seis factores especiales: fomento de la actividad física, masoterapia, 
Revista Iberoamericana

de las Ciencias de la Salud

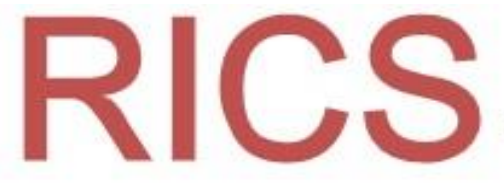

musicoterapia, rehabilitación, actividades lúdicas y aromaterapia, los cuales se explican a continuación.

\section{Fomento de la actividad física}

Este se define como "cualquier movimiento corporal intencional realizado con los músculos esqueléticos, que resulta en un gasto de energía y en una experiencia personal, y nos permite interactuar con los seres y el ambiente que nos rodea" (Devís, 2000, citado por Ceballos, Álvarez y Medina, s. f., p. 3). Sin embargo, en el caso de los adultos mayores, Gil, Ramos, Marín y López (2012) comentan que estos, cuando se mantienen inactivos, pierden sus capacidades fundamentales de resistencia, fortalecimiento, equilibrio y flexibilidad, lo cual podría evitarse con algún tipo de activación física que permita mantener la independencia y reducir la posibilidad de adquirir una enfermedad no transmisible. Al respecto, Ceballos et al. (s. f.) explican que los beneficios de la activación física son inmuebles, ya que se puede aumentar el bienestar general, mejorar la salud física y psicológica general, conservar una vida independiente, ayudar a controlar trastornos específicos (p. ej., estrés, obesidad) y algunas enfermedades (p. ej., diabetes, osteoporosis), así como contribuir a reducir al mínimo las consecuencias de ciertas discapacidades o cambiar la perspectiva estereotipada de la vejez.

\section{Masoterapia}

La masoterapia es definida por Chavez y d'Hyder (citado en d'Hyder, C. y Gutiérrez, L. 2014) como: "Tratamiento mediante el masaje, considerado como toda técnica manual o mecánica que moviliza de forma metódica los tejidos con fines terapéuticos, preventivos, higiénicos, estéticos o deportivos" (p. 743). Esta es una actividad que agrada mucho a las personas mayores, ya que estimula la relajación de los músculos que carecen de actividad física debido al sedentarismo. El masaje geriátrico es un complemento efectivo y específico en el proceso de la vejez, y se genera por medio del contacto sensible y sutil, con técnicas y manipulaciones que estimulan los procesos fisiológicos y orgánicos para mejorar su 
Revista Iberoamericana

de las Ciencias de la Salud

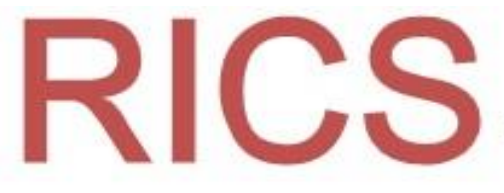

funcionamiento, lo cual mejora la calidad de vida del usuario (González, González, Chacón y Torres, 2013). Este se sustenta en manipulaciones técnicas y sistemáticas, basadas en conceptos científicos que buscan estimular el tejido blando de los órganos, especialmente mediante la aplicación de estiramientos y compresiones rítmicas de forma relajante y terapéutica (Rodríguez, Bustos, Amariles y Rodríguez, 2002).

\section{Musicoterapia}

La musicoterapia, según Bravo, Cabañas, Díez, Gamarra y Villarta (s. f.), puede ser definida desde el punto de vista científico y terapéutico:

Desde el punto de vista científico: la musicoterapia es una especialización científica que se ocupa del estudio e investigación del complejo sonido-ser humano, sea el sonido musical o no, tendiente a buscar los elementos diagnósticos y los métodos terapéuticos del mismo. (p. 4).

Desde el otro punto de vista: es una disciplina paramédica, que utiliza en sonido, la música y el movimiento para producir efectos regresivos y abrir canales de comunicación con el objetivo de emprender a través de ellos el proceso de entrenamiento y recuperación del paciente para la sociedad (p. 5).

La música se ha integrado a las actividades propias de la gerontología, dado el poder curativo que puede generar en el ser humano, de ahí que los gerontólogos la consideren como parte de la intervención gerontológica. Sobre este elemento, González, D. (2014) indica que en el campo psicológico se ha determinado que la música no solo es capaz de facilitar la expresión de sentimientos y emociones, sino que también puede reducir el estrés, calmar el dolor y estabilizar el estado de ánimo. Por tal motivo, en el ámbito terapéutico se ha empleado la música para tratar estados de ansiedad, depresión, estrés, hipertensión, psicosis, autismo y trastornos del comportamiento.

Chica (6 de septiembre de 2015) ha identificado tres beneficios que la musicoterapia aporta a las personas en cuanto a lo cognitivo, lo físico y lo socioemocional; el primero se relaciona con la ayuda que puede ofrecer en el aprendizaje, la orientación en la realidad, la 
capacidad de atención y concentración, así como para mantener u optimizar las habilidades verbales y de comunicación. El beneficio físico se relaciona con la contribución que brinda a la movilidad de las articulaciones, la fuerza del músculo, la relajación, así como para la reducción de la agitación y los niveles de ansiedad. Por último, el tercer beneficio se vincula con el impacto que tiene en la interacción y la comunicación social, ya que reduce y previene el aislamiento, y eleva las habilidades sociales y la autoestima. De hecho, escuchar música alegre mejora el humor.

\section{Rehabilitación}

El sentirse bien contribuye a una percepción más optimista de la vida e incide en mejorar la calidad de vida de las personas; por ello, es importante cuidar la salud desde las etapas iniciales del desarrollo humano, Chavez, D., y d'Hyder, C. (citado en d'Hyder, C. y Gutiérrez, L. 2014)

La World Health Organization (WHO) ha recomendado un modelo para abordar los problemas geriátricos que desarrollan limitaciones en la función física y psicológica y éste ha sido adaptado para la clasificación internacional de funcionalidad, discapacidad y salud (ICF) que incluye las áreas físicas, psicológicas, nivel de independencia, relaciones sociales, ambiente y aspectos trascendentales. (p. 734)

Eso es precisamente lo que se considera en un plan de intervención gerontológica, atención integral de la persona de edad, con la intención de mantener su independencia el mayor tiempo posible de su vida.

\section{Actividades lúdicas}

Las actividades lúdicas se relacionan con el entretenimiento, la diversión y los juegos que se realizan en el tiempo libre. Estas permiten salir de la rutina diaria, relajarse y evitar el estrés, entre otros beneficios físicos y psicológicos, lo que incide en la calidad de vida de la 
persona mayor porque le permite disminuir sus sentimientos de soledad, mejorar el estado de ánimo y la motivación, favorecer un funcionamiento psicomotriz adecuado e incrementar los niveles de autoestima.

\section{Aromaterapia}

Según Sierra (2010), la aromaterapia es la rama de la herbolaria que usa los componentes volátiles de las plantas para obtener efectos terapéuticos; esta se considera como un tratamiento alternativo para combatir los desórdenes físicos, mentales y emocionales a través de aceites de esencias de las plantas. En este sentido, Palomo (2005) expresa que los aromas brindan importantes efectos en la vida del ser humano, pues le ayudan a conseguir el equilibrio emocional y físico. Esta es una actividad que agrada mucho a las personas mayores porque les brinda momentos de relajación y paz.

\section{Método}

El presente trabajo se sustentó en el enfoque cuantitativo, con un alcance exploratorio y con un diseño experimental, pues se empleó una preprueba y una posprueba en un solo grupo. La pregunta de investigación planteada fue la siguiente: ¿cuáles son los beneficios que aporta la intervención gerontológica en la salud de los residentes de un establecimiento privado de asistencia social permanente?

Las conjeturas iniciales fueron estas: después de una intervención gerontológica, los residentes se encuentran motivados para realizar actividad física, mejorar su postura y socializar sus experiencias.

En tal sentido, el objetivo establecido fue identificar los beneficios que aporta la intervención gerontológica en la salud de los residentes de un establecimiento privado de asistencia social permanente. Para ello, se conformó un equipo de colaboradores (voluntarios) integrado por ocho estudiantes de la licenciatura en Gerontología, los cuales brindaron una atención personalizada a cada una de las personas mayores de la mencionada 
Revista Iberoamericana de las Ciencias de la Salud

residencia geriátrica. El análisis de los datos se realizó de manera descriptiva mediante el uso de histogramas y gráficas de líneas.

\section{Lugar}

Se eligió un establecimiento privado de asistencia social permanente ubicado en la ciudad de San Francisco de Campeche, Campeche (México). Sobre estos lugares, cabe destacar que habitualmente se les conoce como asilo, residencia geriátrica, hogar de ancianos o centro gerontológico; sin embargo, en esta investigación se ha usado la expresión establecimiento de asistencia social permanente tomando como base la Norma Oficial Mexicana NOM-031-SSA3-2012, específicamente en el apartado 4.7 (Secretaría de Salud, 2012), donde se establece lo siguiente:

[Se llama] establecimiento de asistencia social permanente a todo aquel lugar que independientemente de su denominación o régimen jurídico otorga atención integral permanente para personas adultas y adultas mayores, que cuentan con características especiales de atención, donde se proporcionan servicios de prevención de riesgos, atención y rehabilitación, incluyen alojamiento, alimentación, vestido, atención médica, social y psicológica, actividades culturales, recreativas y ocupacionales (párr. 21).

Este es el término que se encuentra considerado en la norma oficial, sin embargo, en la observación empírica se aprecia que es poco conocido por los profesionales y por la sociedad en general, son más comunes los términos de: asilo de ancianos, hogar de ancianos, centro geriátrico o residencia geriátrica, por mencionar algunos, siendo importante la difusión de la terminología correcta. 


\section{Población y muestra}

La muestra seleccionada fue no probabilística. Los criterios de inclusión fueron los siguientes: sexo indistinto, mayor de 60 años de edad, sin problemas psiquiátricos graves y que desearan participar en las actividades del proyecto. El establecimiento contaba con once huéspedes; sin embargo, al momento de iniciar el proyecto se excluyeron a tres de ellos por las siguientes causas: persona fallecida, persona con diagnóstico psiquiátrico que no se encontraba disponible y persona con 45 años de edad. La muestra, por tanto, fue de ocho huéspedes (cuatro mujeres y cuatro hombres), de los cuales tres hombres y una mujer usaban silla de ruedas.

\section{Instrumentos}

En este estudio se aplicaron las siguientes cinco escalas de valoración: la escala Fumat para la evaluación objetiva de la calidad de vida de usuarios de servicios sociales (Verdugo, Gómez y Arias, 2009); el miniexamen del estado mental (minimental de Folstein) para establecer la presencia de probable deterioro cognoscitivo; la escala de depresión geriátrica (GDS) para detectar la sintomatología depresiva; el índice de independencia en las actividades básicas de la vida diaria de Katz para establecer el grado de dependencia de los individuos mayores de 60 años en las actividades básicas de la vida diaria, y la evaluación de estabilidad de la marcha y equilibrio (Tinetti) para determinar el riesgo de caídas mediante el análisis de la estabilidad en la marcha y el equilibrio de la persona adulta mayor.

\section{Plan de tratamiento}

Se realizaron once sesiones: una para la presentación y aplicación de los instrumentos y diez para el tratamiento. Cada una de estas tuvo una duración aproximada de dos horas (de 4 a 6 de la tarde) (tabla 1). Las actividades se realizaron en el marco de la ética y siguiendo en todo momento los principios de autonomía, beneficencia, justicia y respeto a la persona, 
para lo cual se preparó una versión ejecutiva del protocolo y se entregó a través de oficio al establecimiento donde se realizó el estudio.

Los cinco instrumentos se aplicaron en la sesión diagnóstica (pretest) y en la última sesión (postest). Todas las sesiones iniciaron con un módulo de 30 minutos de ejercicios de calentamiento, fuerza, resistencia y relajación; posteriormente, se agregó un segundo módulo de la sesión 1 a la 7, así como un tercer módulo en las sesiones 8, 9 y 10. A continuación, se describe lo trabajado en cada una:

- El segundo módulo se trabajó de la siguiente forma: sesión 1 y 2, M2 (módulo dos), ejercicios de atención.

- Sesión 3, M2, musicoterapia.

- Sesión 4, M2, karaoke.

- Sesión 5 y 6, M2, ejercicios de rehabilitación y masoterapia.

- Sesión 7, M2, ejercicios de coordinación con globos.

- En esta sesión 8 se integró un módulo más, es decir, se trabajó con tres módulos:

Sesión 8, M2, ejercicios de rehabilitación y masoterapia. M3 (módulo tres), aromaterapia.

- Sesión 9, M2, ejercicios de rehabilitación y masoterapia, M3, ejercicio de motricidad fina: jenga.

- Sesión 10, M2, lotería, M3, boliche (en esta última sesión se aplicaron las cinco escalas como postest). 


\section{Revista Iberoamericana de las Ciencias de la Salud}

Tabla 1. Protocolo del tratamiento aplicado

\section{Protocolo del tratamiento aplicado}

\begin{tabular}{|c|c|}
\hline $\begin{array}{l}\text { ción } \\
\text { apia }\end{array}$ & $\begin{array}{l}\text { Se realizaron movimientos pasivos forzados (manipulación de alguna parte del cuerpo, lo } \\
\text { cual se realizó en parejas, llevando al máximo el límite de movilidad anatómica de alguna } \\
\text { parte del cuerpo sin causar dolor ni violencia en el movimiento). Se inició la rehabilitación } \\
\text { con la extensión y flexión de los miembros superiores, especialmente las manos y los } \\
\text { dedos, ya que se observó que la mitad de los pacientes tenían empuñada la mano. Luego } \\
\text { se pasó a los miembros inferiores, pues se apreció que la dorsiflexión y la flexión plantar } \\
\text { se encontraban limitadas; además, los pies se hallaban edematizados por estar sentados la } \\
\text { mayor parte del día en sus sillas de ruedas. Para finalizar la sesión se trabajó con la } \\
\text { masoterapia en los músculos del cuello y la espalda. }\end{array}$ \\
\hline $\begin{array}{l}\text { Actividad con } \\
\text { globo }\end{array}$ & $\begin{array}{l}\text { Para trabajar la fuerza y los movimientos activos se desarrollaron actividades con un globo } \\
\text { de la siguiente forma: se colocó a un lado de su cabeza para emplear toda la fuerza } \\
\text { sosteniendo el globo. Después se colocó entre el antebrazo y el brazo, los cuales fueron } \\
\text { usados para sostenerlo en su lugar. Luego, para trabajar las manos y las muñecas, se les } \\
\text { pidió que con cada uno de sus dedos hundieran el globo, y después que lo tomaran con la } \\
\text { muñeca para que también lo presionaran. Para ejercitar los miembros inferiores se les } \\
\text { solicitó que colocaran el globo entre las piernas y luego que ejercieran presión. } \\
\text { Posteriormente, se enfocó el trabajo en las rodillas y después en el área de las pantorrillas } \\
\text { (en cada una de las posiciones ejercieron presión } 10 \text { veces). Finalmente, se les pidió que } \\
\text { pisaran el globo con la mayor fuerza posible. Esta sesión concluyó con ejercicios de } \\
\text { relajación, a través de respiraciones largas y profundas. Los participantes indicaron que no } \\
\text { sintieron ninguna molestia durante los ejercicios y que les habían gustado las actividades. }\end{array}$ \\
\hline $\begin{array}{l}\text { Ejercicio de } \\
\text { coordinación }\end{array}$ & $\begin{array}{l}\text { Nuevamente se emplearon globos, los cuales debían pasar a sus compañeros en la dirección } \\
\text { que se le indicaba (izquierda o derecha); después, con una mano, sostenían el globo y con } \\
\text { la otra se tocaban alguna parte del cuerpo. Esta activad procuró fomentar la atención hacia } \\
\text { las indicaciones señaladas. }\end{array}$ \\
\hline Aro & $\begin{array}{l}\text { Para esta se utilizaron bastones aromáticos. En concreto, se les pidió que cerraran los ojos } \\
\text { y con un tono de voz suave y tranquila se les fue guiando para llevarlos mentalmente a un } \\
\text { lugar de paz y tranquilidad. }\end{array}$ \\
\hline $\begin{array}{l}\text { Destreza } \\
\text { agilidad }\end{array}$ & $\begin{array}{l}\text { Esta actividad fue útil para trabajar la motricidad fina. Para desarrollarla se empleó un } \\
\text { juego conocido con el nombre de jenga, el cual consiste en acomodar rectángulos de } \\
\text { madera para formar una torre, los cuales posteriormente se van extrayendo para seguir } \\
\text { construyendo en la parte superior hasta que la estructura se derrumbe. }\end{array}$ \\
\hline
\end{tabular}

Fuente: Elaboración propia 


\section{Material}

Para el ejercicio de calentamiento, fuerza y resistencia se usaron diez botellas de plástico de $500 \mathrm{ml}$ (una para cada huésped), las cuales fueron llenadas con arena. Asimismo, se empleó un juego de boliche realizado con botellas pintadas por el equipo de voluntarios. Igualmente, se trabajó con el jenga y con un juego de lotería donado por el equipo. También se suministraron pañales desechables, guantes, cubrebocas y material de curación.

\section{Resultados}

En la tabla 2 se presenta el avance obtenido en las sesiones según se fue desarrollando el plan de intervención.

Tabla 2. Principales resultados del tratamiento aplicado

\begin{tabular}{|c|c|c|c|}
\hline $\begin{array}{l}\mathrm{N} .^{\circ} \mathrm{de} \\
\text { sesión }\end{array}$ & $\begin{array}{c}\text { Número } \\
\text { de PM }\end{array}$ & Actividad & Observaciones \\
\hline $\begin{array}{l}\text { Diagnó } \\
\text { stico }\end{array}$ & 8 & $\begin{array}{l}\text { 1. Se aplicaron las escalas } \\
\text { de valoración (pretest) }\end{array}$ & $\begin{array}{l}\text { Se observa a los huéspedes un poco desorientados y } \\
\text { apáticos con nuestra presencia. }\end{array}$ \\
\hline 1 & 6 & $\begin{array}{l}\text { 1. Ejercicios de } \\
\text { calentamiento, fuerza, } \\
\text { resistencia y relajación. } \\
\text { 2. Ejercicios de atención y } \\
\text { concentración. }\end{array}$ & $\begin{array}{l}\text { Debilidad, sin fuerza en brazos y poca coordinación en } \\
\text { manos y pies. } \\
\text { Se observa a los huéspedes un poco desorientados y } \\
\text { apáticos con nuestra presencia. } \\
\text { Colorear dibujos y números, identificar letras; algunos } \\
\text { adultos mayores fueron capaces de realizar la actividad } \\
\text { sin ayuda; otros sí necesitaron apoyo. }\end{array}$ \\
\hline 2 & 5 & $\begin{array}{l}\text { 1. } \begin{array}{l}\text { Ejercicios de } \\
\text { calentamiento, fuerza, } \\
\text { resistencia y relajación }\end{array} \\
\text { 2. Ejercicios de atención y } \\
\text { concentración }\end{array}$ & $\begin{array}{l}\text { Se observa a los pacientes un poco incomodos; algunos } \\
\text { comentan que lo sentían pesado, por lo que se } \\
\text { disminuyen las repeticiones. } \\
\text { En el ejercicio de atención se observa diálogo entre los } \\
\text { huéspedes y el equipo; incluso conversan un poco sobre } \\
\text { su vida. }\end{array}$ \\
\hline 3 & 6 & $\begin{array}{l}\text { 1. } \begin{array}{l}\text { Ejercicios de } \\
\text { calentamiento, fuerza, } \\
\text { resistencia y relajación. }\end{array} \\
\text { 2. Musicoterapia. }\end{array}$ & $\begin{array}{l}\text { Los huéspedes se muestran un poco más accesibles a las } \\
\text { indicaciones recibidas al realizar el ejercicio. }\end{array}$ \\
\hline 4 & 6 & $\begin{array}{l}\text { 1. Ejercicios de } \\
\text { calentamiento, fuerza y } \\
\text { resistencia, y relajación }\end{array}$ & $\begin{array}{l}\text { En el karaoke, al principio, se observaba desinterés } \\
\text { hacia la actividad porque les daba pena cantar, pero } \\
\text { después de que nos observaron cantar y poner canciones } \\
\text { de su gusto, su interés creció y poco a poco comenzaron } \\
\text { a cantar con nosotros. Algunos huéspedes cantaron } \\
\text { solos. } \\
\text { Al final, se observó un gusto por la actividad, la cual les } \\
\text { recordó bonitos momentos por las letras de las } \\
\text { canciones. }\end{array}$ \\
\hline
\end{tabular}




\section{Revista Iberoamericana de las Ciencias de la Salud}

\begin{tabular}{|c|c|c|c|}
\hline 5 & 4 & 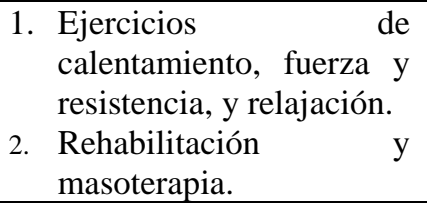 & $\begin{array}{l}\text { Se observa a los huéspedes más tranquilos, accesibles y } \\
\text { de un mejor humor; se siente una sensación más } \\
\text { agradable en la estancia. }\end{array}$ \\
\hline 6 & 5 & 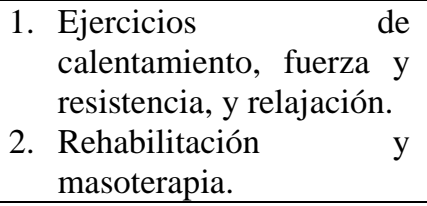 & $\begin{array}{l}\text { Los huéspedes ya nos esperaban; se observa un } \\
\text { ambiente tranquilo y de confianza. }\end{array}$ \\
\hline 7 & 7 & $\begin{array}{l}\text { 1. Ejercicios de } \\
\text { calentamiento, fuerza y } \\
\text { resistencia, y relajación. } \\
\text { 2. Ejercicios de } \\
\text { coordinación: actividad } \\
\text { con globos. }\end{array}$ & $\begin{array}{l}\text { Los huéspedes refieren no sentir dolor o molestia, se } \\
\text { muestran muy satisfechos y contentos con nuestra } \\
\text { presencia y las actividades que llevamos realizando con } \\
\text { ellos. } \\
\text { Se aprecian mejorías en los huéspedes en cuanto al } \\
\text { estado de humor, la accesibilidad a las actividades, la } \\
\text { relación con nosotros y la amplitud en su rango de } \\
\text { movilidad. }\end{array}$ \\
\hline 8 & 6 & 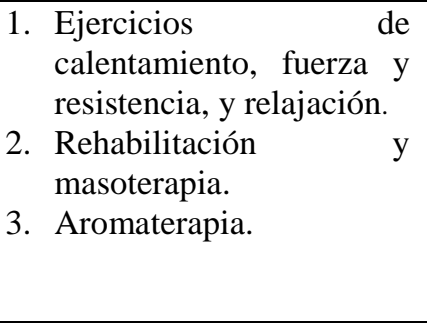 & $\begin{array}{l}\text { Ya no se quejan por el peso de la botella, sus } \\
\text { movimientos son más suaves, ha disminuido la rigidez } \\
\text { en sus miembros superiores, así que ya van coordinados } \\
\text { con los movimientos. } \\
\text { Durante la rehabilitación, se observa mayor rango de } \\
\text { movimiento, sin presencia de dolor; se aprecia una } \\
\text { mejor apariencia en cada uno de ellos, un aumento de } \\
\text { ánimo y una mejor motricidad. }\end{array}$ \\
\hline 9 & 6 & $\begin{array}{l}\text { 1. Ejercicios de } \\
\text { calentamiento, fuerza, } \\
\text { resistencia y relajación. } \\
\text { 2. Rehabilitación } \\
\text { masoterapia. } \\
\text { 3. Jenga }\end{array}$ & $\begin{array}{l}\text { El nivel de confianza y vínculo de amistad ha crecido. } \\
\text { Al utilizar las pesas se notan una mayor fuerza y } \\
\text { resistencia; aumenta el número de movimientos, y se } \\
\text { pasa de } 8 \text { a } 10 \text { repeticiones sin observar molestia. } \\
\text { En este juego, se puede observar que los huéspedes se } \\
\text { divirtieron mucho y lo comprendieron con sus reglas; } \\
\text { pasan una tarde muy agradable, aunque un poco } \\
\text { angustiosa por la torre cuando observaban que su } \\
\text { compañero corría el riesgo de tirarla. Su motricidad fina } \\
\text { ha mejorado considerablemente, ya que al principio } \\
\text { tenían las manos en forma de puño, pero con las sesiones } \\
\text { esto ha disminuido y se observa mayor agilidad en sus } \\
\text { movimientos. }\end{array}$ \\
\hline 10 & 5 & $\begin{array}{l}\text { 1. Ejercicios de } \\
\text { calentamiento, fuerza y } \\
\text { resistencia, y relajación. } \\
\text { 2. Ejercicio de habilidad y } \\
\text { destreza: boliche. } \\
\text { 3. Ejercicio de atención y } \\
\text { concentración: lotería. } \\
\text { 4. Se aplicaron las escalas } \\
\text { de valoración (postest). }\end{array}$ & $\begin{array}{l}\text { Las repeticiones han aumentado de } 10 \text { a } 13 \text {, sin observar } \\
\text { ninguna molestia; el rango de movilidad y fuerza ha } \\
\text { aumentado considerablemente. } \\
\text { Han dejado de sentir el peso, resisten todos los } \\
\text { ejercicios. } \\
\text { Se divirtieron mucho y se observa que tienen una } \\
\text { puntería considerable. }\end{array}$ \\
\hline
\end{tabular}

Fuente: Elaboración propia 
Revista Iberoamericana de las Ciencias de la Salud

A partir de lo indicado en la tabla 2 se puede afirmar que las personas mejoraron aspectos relacionados con la flexibilidad, la movilidad, la atención, la concentración y la socialización. Por otra parte, los principales resultados obtenidos según cada una de las escalas aplicadas fueron los siguientes:

- Minimental de Folstein: Se determinó que todos los hombres participantes tienen deterioro cognitivo, mientras que en todas las mujeres participantes hay indicios de demencia.

- Katz: Todos los hombres tienen una incapacidad leve, mientras que todas las mujeres tienen incapacidad moderada.

- Tinetti: Todos los participantes tienen problemas de marcha y equilibrio.

- GDS: Toda la población participante obtuvo valores normales.

- Fumat: De los 100 puntos que se puede conseguir con esta escala, $62.5 \%$ de los participantes considera como satisfactoria la percepción de la calidad de vida en la residencia geriátrica (figura 1 ).

Figura 1. Resultados obtenidos en la escala Fumat

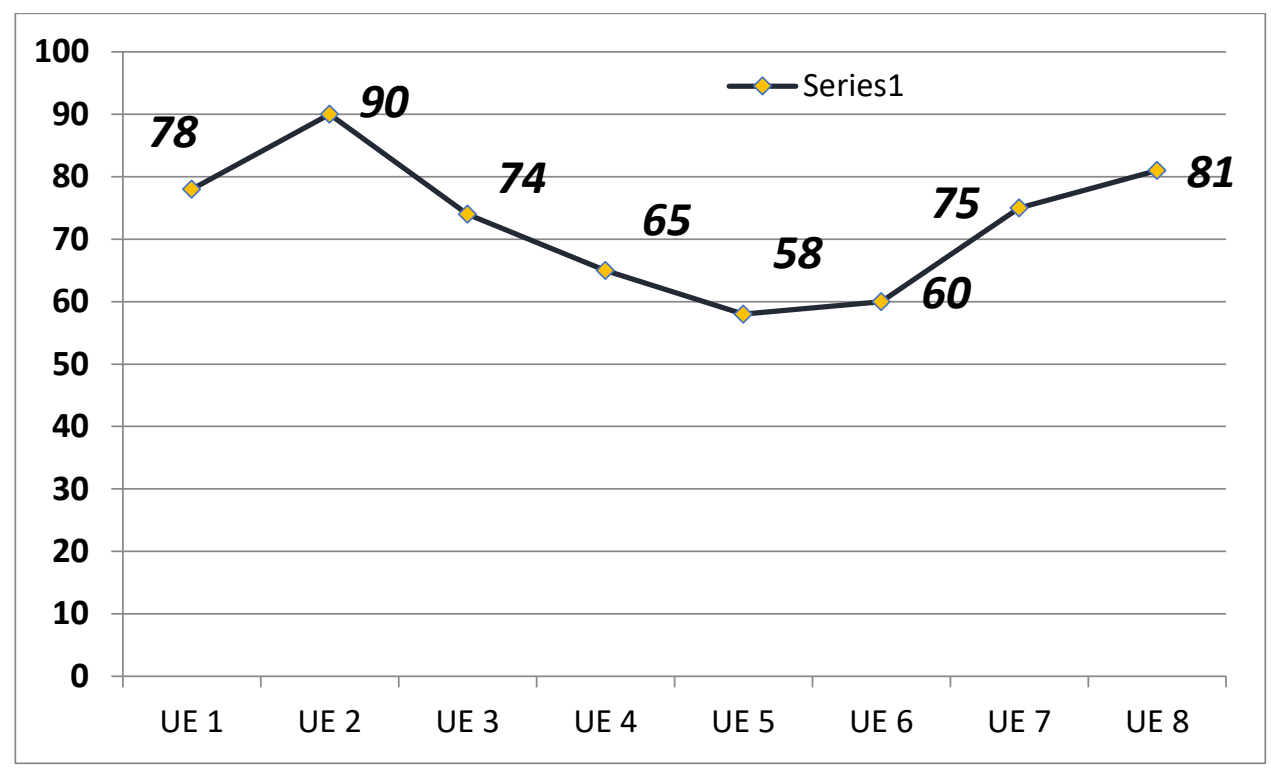

Fuente: Elaboración propia 
Sin embargo, vale destacar que no se observa una diferencia significativa en las escalas aplicadas antes y después del plan de tratamiento (figura 2), aunque la observación empírica recabada en las sesiones evidenció una evolución favorable de los participantes.

Figura 2. Resultados obtenidos en el pretest y en el postest

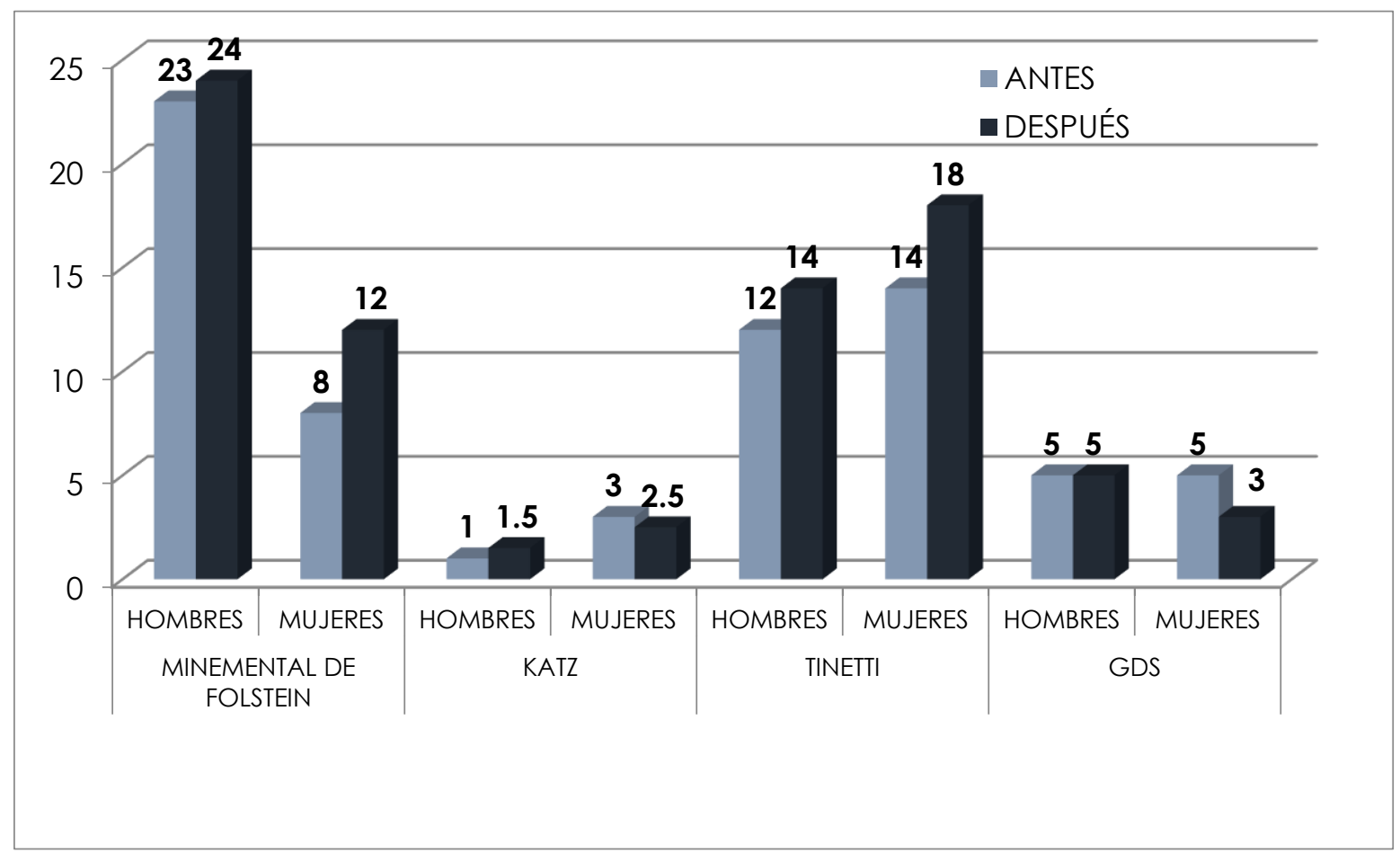

Fuente: Elaboración propia

\section{Discusión}

La principal limitante encontrada en este estudio fue que no se logró mantener el número de ocho participantes en todas las sesiones debido a sus características pluripatológicas (en ocasiones se sentían indispuestos y se mantenían en sus habitaciones); de los cuatro hombres, solo dos participaron en la mayoría de las actividades, a diferencia de las mujeres, quienes se mantuvieron hasta el final. 
La intervención gerontológica, por otra parte, demostró favorecer la salud de las personas mayores, ya que representa el tratamiento no farmacológico complementario que necesitan para mejorar su estado.

Asimismo, y después de analizar los resultados conseguidos, se puede afirmar que este estudio es coherente con dos de los objetivos del desarrollo sostenible propuestos por la Organización de Naciones Unidas (ONU, s. f.), es decir, salud y bienestar, y reducción de las desigualdades, pues la intervención gerontológica procura fomentar la independencia de la persona mayor a través del mejoramiento de sus condiciones.

Igualmente, se confirma lo señalado por la OMS, pues para mantener la salud en las personas mayores se deben tomar en cuenta los factores tanto individuales como ambientales; los primeros, como ya se indicó, se vinculan con el cuidado propio de la salud que cada persona realiza mediante su comportamiento y su adaptación a los cambios de su edad; mientras que los factores ambientales se relacionan con las responsabilidades que tienen los establecimientos de asistencia social permanente, los cuales deben atender y procurar la socialización de las personas mayores que allí residen.

Por último, se confirma lo expuesto por Mena et al. (2012), quienes apuntan que "para poder desarrollar las intervenciones, el gerontólogo debe utilizar una serie de instrumentos especializados que aporten los datos necesarios para analizar y planear dichas intervenciones para la atención multidimensional de la persona adulta mayor" (p. 8). En este sentido, el plan de tratamiento de este estudio siguió la propuesta de aplicación de escalas de valoración y protocolos de intervención, y se comprobaron los beneficios que se derivan de ellos.

\section{Conclusiones}

Los establecimientos privados de asistencia social permanente necesitan del apoyo de las instituciones educativas del área de la salud para que sean considerados como sedes de la práctica clínica y del servicio social, lo cual daría como resultado una mejor atención a los huéspedes, pues la atención de las personas ancianas demanda elevados gastos que las cuotas mensuales no suelen cubrir en su totalidad. 
Asimismo, se debe insistir en que el tratamiento no farmacológico resulta indispensable para estas personas, por lo que se debe fomentar una cultura de intervención gerontológica que permita alcanzar resultados satisfactorios en todos los establecimientos de asistencia social permanente.

Igualmente, se debe prever que el trabajo con personas adultas mayores pluripatológicas representa un reto para el profesional de la salud, el cual debe tener paciencia y conocimiento para diseñar, aplicar y evaluar los resultados conseguidos con un plan de intervención, para lo cual se requiere colaboración interdisciplinaria.

\section{Agradecimientos}

Queremos expresar nuestra gratitud y reconocimiento al centro gerontológico privado de la ciudad de San Francisco de Campeche, por permitirnos colaborar con el trabajo que realizan con sus huéspedes y por las facilidades otorgadas en el desarrollo de este estudio. Asimismo, agradecemos al equipo de estudiantes de la licenciatura en Gerontología que colaboró en cada una de las sesiones: Shirley, Alexander, David, Luis, Raymundo, Verónica, Luis Enrique y Lourdes Maricruz; gracias por el entusiasmo, disponibilidad y empatía demostrada en cada sesión del plan de actividades. 
Revista Iberoamericana

de las Ciencias de la Salud

ISSN: $2395-8057$

\section{Referencias}

Bravo, M., Cabañas, A., Díez, F., Gamarra, M. y Villarta, E. (s. f.). Musicoterapia. Recuperado de https://previa.uclm.es/profesorado/ricardo/AlumnosEE/Musicot_Prim06.doc.

Ceballos, O., Álvarez, J. y Medina, R. (s. f). Actividad física y calidad de vida en adultos mayores. Editorial El Manual Moderno. Recuperado de http://eprints.uanl.mx/4476/1/Capitulos\%20de\%20libro.pdf.

Chica, A. (6 de septiembre de 2015). Los beneficios de la musicoterapia para el bienestar de los mayores Recuperado de http://geriatricarea.com/los-beneficios-de-lamusicoterapia-para-el-bienestar-de-los-mayores/.

d’Hyder, C. y Gutiérrez, L. (2014). Geriatría. Capítulo 65. Generalidades en la rehabilitación geriátrica. De la autoría de Chavez, D. y d'Hyder, C. 3ª Edición. Manual Moderno. 944 páginas.

Gil, P., Ramos, P., Marín, J. y López, J. (2012). Guía de ejercicio físico para mayores. Editorial Grupo ICM Comunicación. Recuperado de https://www.segg.es/media/descargas/Acreditacion\%20de\%20Calidad\%20SEGG/Ce ntrosDia/GU\%C3\%8DA\%20DE\%20EJERCICIO\%20F\%C3\%8DSICO\%20PARA\% 20MAYORES.pdf.

González, D. (2014). La musicoterapia como herramienta para reducir el nivel de depresión en adultos mayores deprimidos e institucionalizados en un hogar de ancianos privado (trabajo de grado). Guatemala: Universidad Rafael Landívar. Recuperado de http://recursosbiblio.url.edu.gt/tesiseortiz/2014/05/42/Gonzalez-Danilo.pdf.

González, M., González, I., Chacón, P. y Torres, M. (2013). Beneficios del masaje geriátrico en la salud de los ancianos de la fundación Granitos de Paz durante el segundo período del 2012 y primer período del 2013. Corporación Universitaria Rafael Núñez. Recuperado de http://siacurn.app.curnvirtual.edu.co:8080/xmlui/bitstream/handle/123456789/626/BE 
Revista Iberoamericana de las Ciencias de la Salud

NEFICIOS\%20DEL\%20MASAJE\%20GERI\%C3\%81TRICO\%20EN\%20LA\%20SA LUD\%20DE\%20LOS\%20ANCIANOS\%20DE\%20LA\%20FUNDACI\%C3\%93N\%2 OGRAN_20130614122736957.pdf?sequence=1.

Instituto de Mayores y Servicios Sociales (Imserso) (2015). Nuevo modelo de intervención gerontológica en el Sistema Nacional de Desarrollo Integral de la Familia de México (SNDIF). Recuperado de http://www.imserso.es/InterPresent2/groups/imserso/documents/binario/enlace28_n mmexico_41_43.pdf.

Instituto Nacional de Estadística y Geografía (Inegi) (2003). La evolución de los hogares unipersonales 1990-2000. Recuperado de http://internet.contenidos.inegi.org.mx/contenidos/Productos/prod_serv/contenidos/ espanol/bvinegi/productos/historicos/2104/702825497484/702825497484_1.pdf.

Instituto Nacional de Estadística y Geografía (Inegi) (2015). Encuesta intercensal 2015. Recuperado de http://www.beta.inegi.org.mx/temas/estructura/.

Instituto Nacional de Estadística y Geografía (Inegi) (2017). Porcentaje de hogares $\begin{array}{llll}\text { unipersonales } & \text { México. Recuperado de }\end{array}$ http://www.beta.inegi.org.mx/app/buscador/default.html?q=\%C3\%ADndice+de+sol edad\#tabMCcollapse-Indicadores.

Mena, R., Lozano, M., Arévalo, A., Archundia, R., Olivares, A., Chaparro, É. y Esperón, R. (2012). Principios del abordaje gerontológico en la persona adulta mayor e intervenciones básicas. Programa de salud en el adulto y anciano de la Secretaría de Salud. Recuperado de http://seminarioenvejecimiento.unam.mx/Publicaciones/libros/principios_abordaje.pd $\underline{\mathrm{f}}$

Organización de las Naciones Unidas (ONU) (s. f.). Objetivos de desarrollo sostenible. Recuperado de http://www.onu.org.mx/agenda-2030/objetivos-del-desarrollosostenible/. 
Revista Iberoamericana

de las Ciencias de la Salud

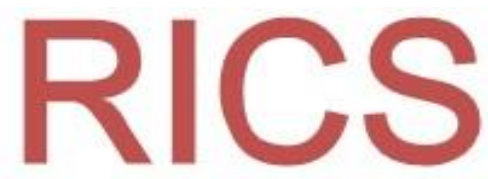

ISSN: $2395-8057$

Organización Mundial de la Salud (OMS) (2015). Envejecimiento y salud. Recuperado de http://www.who.int/ageing/ageing-infographic-2015-es.pdf?ua=1

Palomo, M. (2005). Aromaterapia. Guatemala: Universidad de San Carlos de Guatemala. Recuperado de http://biblioteca.usac.edu.gt/tesis/07/07_1776.pdf.

Rodríguez, J., Bustos, J., Amariles, C. y Rodríguez, H. (2002). El masaje terapéutico en lesiones musculares producidas por traumas de tejidos blandos. Revista Médica de Risaralda, $8(2)$ Recuperado de https://revistas.utp.edu.co/index.php/revistamedica/article/view/8137.

Sanjoaquín, A., Fernández, E., Mesa, M. y García, E. (2004). Valoración geriátrica integral (capítulo 4) (pp. 59-68). España: Sociedad Española de Geriatría y Gerontología. Recuperado de https://www.segg.es/tratadogeriatria/PDF/S35-05\%2004_I.pdf.

Secretaría de Salud (2012). Norma Oficial Mexicana NOM-031-SSA3-2012. Asistencia social. Prestación de servicios de asistencia social a adultos y adultos mayores en situación de riesgo y vulnerabilidad. Recuperado de http://dof.gob.mx/nota_detalle.php?codigo=5267965\&fecha=13/09/2012.

Sierra, H. (2010). Manual de aromaterapia. Recuperado de http://datelobueno.com/wpcontent/uploads/2014/05/Manual-de-Aromaterapia.pdf.

Verdugo, M., Gómez, A. y Arias, B. (2009). Escala de la calidad de vida en personas mayores: la escala FUMAT. Salamanca (España): Publicaciones del INICO. Recuperado de http://sid.usal.es/idocs/F8/FDO23248/herramientas_4.pdf. 


\begin{tabular}{|c|c|}
\hline Rol de Contribución & Autor (es) \\
\hline Conceptualización & Jaqueline Guadalupe Guerrero Ceh \\
\hline Metodología & Jaqueline Guadalupe Guerrero Ceh (Principal), Lourdes Maricruz Morales (apoyo) \\
\hline Software & $\begin{array}{l}\text { Jaqueline Guadalupe Guerrero Ceh (Principal), Ana Rosa Ca, Valle (apoyo), } \\
\text { Lourdes Maricruz Morales (apoyo) }\end{array}$ \\
\hline Validación & Jaqueline Guadalupe Guerrero Ceh (Principal), Lourdes Maricruz Morales (apoyo) \\
\hline Análisis Formal & Jaqueline Guadalupe Guerrero Ceh (Principal), Lourdes Maricruz Morales (apoyo) \\
\hline Investigación & Jaqueline Guadalupe Guerrero Ceh (Principal), Lourdes Maricruz Morales (apoyo) \\
\hline Recursos & $\begin{array}{l}\text { Jaqueline Guadalupe Guerrero Ceh (Principal), Ana Rosa Can Valle (apoyo), } \\
\text { Lourdes Maricruz Morales (apoyo) }\end{array}$ \\
\hline Curación de datos & Jaqueline Guadalupe Guerrero Ceh (Principal), Ana Rosa Can Valle (apoyo) \\
\hline $\begin{array}{l}\text { Escritura - Preparación del borrador } \\
\text { original }\end{array}$ & Jaqueline Guadalupe Guerrero Ceh \\
\hline Escritura - Revisión y edición & Jaqueline Guadalupe Guerrero Ceh \\
\hline Visualización & Jaqueline Guadalupe Guerrero Ceh \\
\hline Supervisión & Jaqueline Guadalupe Guerrero Ceh (Principal), Lourdes Maricruz Morales (apoyo) \\
\hline Administración de Proyectos & Jaqueline Guadalupe Guerrero Ceh \\
\hline Adquisición de fondos & Jaqueline Guadalupe Guerrero Ceh \\
\hline
\end{tabular}

